\title{
The Roles of The Professional Nurse During The Vaping Crisis
}

\author{
Larider Ruffin* \\ Department of Health Sciences, Stockton University, USA \\ *Corresponding author: Larider Ruffin, Department of Health Sciences, Stockton University, USA.
}

To Cite This Article: Larider Ruffin, The Roles of The Professional Nurse During The Vaping Crisis. Am J Biomed Sci \& Res. 2019 - 6(4). AJBSR. MS.ID.001060. DOI: 10.34297/AJBSR.2019.06.001060.

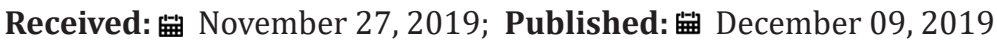

\begin{abstract}
The United States of America experienced a major increase in the number of patients affected by the devastating vaping epidemic. Over 2,200 lung injuries have been reported secondary to vaping. This manuscript seeks to guide the professional nurse to capitalize on the trust earned from the public to advocate for a smoke-free environment, educate patients about the dangers of vaping, and triage patients in a timely manner to help break the vaping crisis.
\end{abstract}

\section{Introduction}

Electronic cigarettes (E-cigarettes) are battery-operated devices that transport a nicotine- containing aerosol or vapor by heating the liquid. The liquid usually contains nicotine, propylene glycol or glycerol, chemicals, and a flavoring agent. Additionally, e-cigarettes are used to vape illicit substances such as cannabis. When the chemicals are heated, they convert to toxic aldehydes that cause lung disorders, inflammation, and upper airway irritation. Some of the flavorings for e-cigarettes contain chemicals that can cause inflammatory obstruction of the bronchioles. This is called bronchiolitis obliterans (popcorn lungs). Bronchiolitis obliterans is an injury to the small airways. The signs and symptoms of bronchiolitis obliterans are cough, dyspnea, wheezing, and fatigue. The symptoms are usually slow and progressive [1-3].

\section{Trends In Vaping}

E-cigarette use increased in high school students in the United States (U.S) from $11.7 \%$ in 2017 to $20.8 \%$ in 2018 . E-cigarette use increased in middle school students in the U.S from 3.3\% in 2017 to 4.9\% in 2018. Approximately 3.62 million middle and high school students were current users of e-cigarettes in 2018 [4]. A large national survey administered annually to students respectively in 8th grade, 10 th grade, and 12th grade found vaping prevalence more than doubled in each of the three grades from 2017 to 2019 [5]. With e-cigarettes marketing campaigns capturing the attention of youth, vaping among middle school and high school students has exceeded cigarette use. Current advertising and health debates about e-cigarette use do not include the negative health effects of nicotine addiction and the vulnerability of young people to nicotine because their brains are in a critical time of development [1].

\section{Vaping Flavored E-Cigarettes}

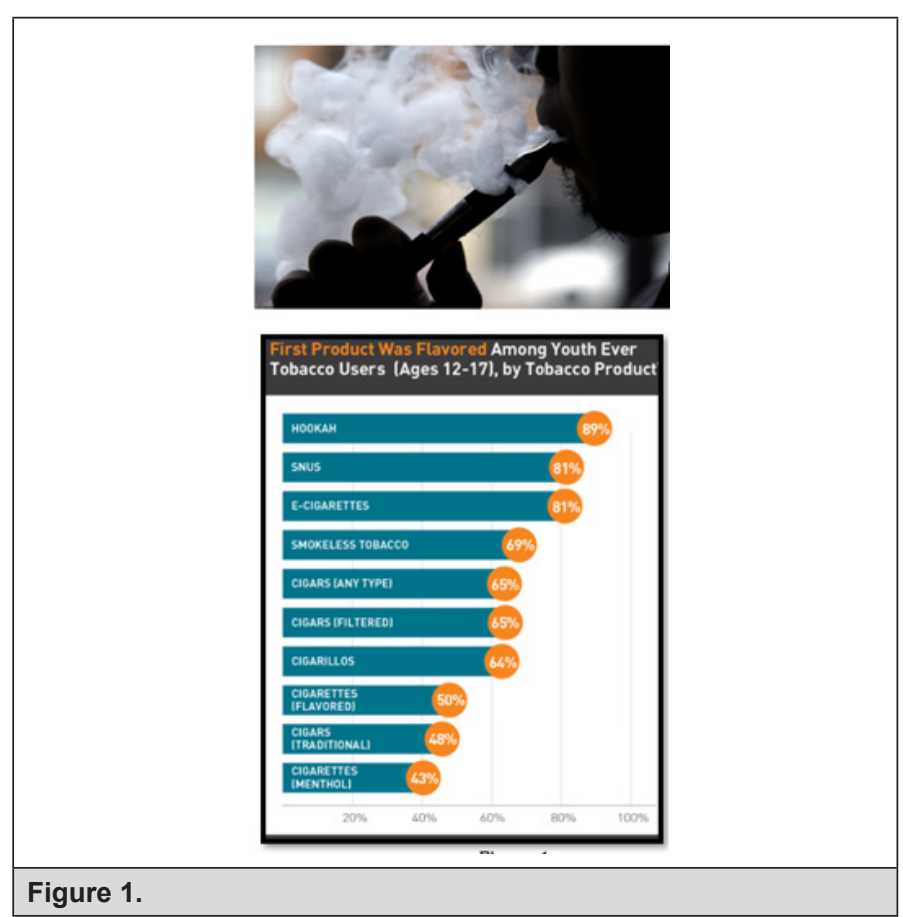


Many tobacco companies claim the flavoring in electronic cigarettes is in food products which consumer ingests daily. While this claim may be truth, food products in the pulmonary system is associated in health hazards. Diacetyl and acetyl propionyl found in flavoring may be safe to eat, data suggest, however, that they are not safe when vaporized and breathed (National Institute for Occupational Safety and Health, CDC).Unfortunately, the youth vaping crisis is directly related to the appeal of flavored e-cigarettes [6]. A recent survey attests $97 \%$ of youth e-cigarette users report using a flavored product in the past month while $70 \%$ cite flavors as the reason for their use. Many teens and pre-teens vape due to flavoring. Unfortunately, there are over 8,000 different types of flavors on the market. The youth used different types of Electronic Nicotine Delivery Systems (ENDS) which include electronic cigarettes to inhale their flavored nicotine (Figure 1).

\section{It's More Than Just Vitamin E}

CDC (2019) identified vitamin E acetate as a chemical of concern among people with e-cigarette, or vaping, product use associated lung injury (EVALI). Vitamin $\mathrm{E}$ is a fat-soluble antioxidant that stops the production of reactive oxygen species formed when fat undergoes oxidation, which, in turn might help prevent or delay chronic diseases associated with free radicals (National Institute of Health, 2019). While patients are being encouraged to keep take vitamins, the tobacco industry used vitamin E acetate as an additive in the production of e-cigarette and other vaping products. Vitamin E acetate resembles tetrahydrocannabinol (THC) oil. The liquid in e-cigarettes may contain nicotine, THC, cannabinoid (CBC) oils, and other substances and additives. THC is the psychoactive mind-altering compound of marijuana that produces the "high" that individuals may experience. When ingested as a vitamin supplement or applied to the skin, vitamin E acetate usually does not cause harm. However, previous research suggests when vitamin $\mathrm{E}$ acetate is inhaled, it may interfere with normal lung functioning [6].

\section{The Outbreak}

As of mid-November 2019, the CDC has investigated samples of bronchoalveolar lavage (BAL) fluid from 29 injured patients represented from 10 States. Vitamin $\mathrm{E}$ acetate has been identified in $100 \%$ of the sample, THC in $82 \%$, while nicotine was identified in $62 \%$ of the sample (CDC, 2019). The investigation continues and spans across nearly all states, involves over 2,000 injured patients. Forty-Seven deaths have been reported in 25 states and the District of Columbia. The age of deceased patients ranged from 17 to 75 year of age with $40 \%$ of cases between the ages of 18 and $24,25 \%$ between 25 and 34 (CDC, 2019).

\section{A Call for Policy Change}

Given the state of the youth e-cigarette epidemic, severe illnesses and deaths linked to electronic cigarette use, the National
Black Nurses Association (NBNA) Ad Hoc Committee on Substance Use Disorders called on all nurses to take action to educate the population about the health hazards of vaping. The NBNA called on the FDA to act quickly to save the youth and young adults.

According to the CDC three areas are most concern:

a) Evidence detailing the long-term and short-term effects of vaping,

b) The risk of nicotine addiction,

c) and distrust that the e-cigarette industry will support regulation given their advertisements targeting the youth (CDC, 2019).

The NBNA called on Congress to pass legislations that ban the sales of flavored e-cigarettes.

\section{The Roles of The Nurse}

The roles of the nurse go far beyond caring for the sick. The nurse should always be willing and ready to apply the systematic guide of the nursing process while advocating for the vulnerable population. The nurse, as the most trusted professional across healthcare, said Milton [7] should advocate at the Federal, State, and local levels by partnering with Legislators and advocate for change. Nurses need to encourage legislation to prevent the sales, marketing, and use of e-cigarettes. During the vaping crisis, if a patient in the community has been vaping and experienced respiratory issues such as a cough, shortness of breath, wheezing, chest pain, gastrointestinal symptoms (abdominal pain, nausea, vomiting), or constitutional symptoms (fever, chills, or weight loss), they should go to the Emergency Department (ED) immediately for further evaluation (Jatlaoui et al.). Patients that vape should tell their primary care provider (PCP) of any of the symptoms immediately for further direction. The role of nurses during the vaping crisis is to be knowledgeable about vaping, advocate for patients, follow institutional protocol, and if EVALI or bronchiolitis obliterans is suspected in the community, patients should be referred to the ED for prompt evaluation. As the most trusted professionals, nurses should support patients to stop smoking and vaping while supporting a smoke-free environment (Schnur, 2019).

Nurses should know that the nicotine in e-cigarettes varies from 0 to $36 \mathrm{mg} / \mathrm{ml}$. Even the so-called nicotine-free products have been shown to contain nicotine, and heating e-liquid which elevates temperatures increases nicotine release with negative effects. When nurses are evaluating patients with respiratory issues, they should ask patients if they have used e-cigarette products or vaped in the last 3 months[8]. If the patients say "Yes," nurses should ask about the substances used (homemade liquid, re-used old cartridges, commercially purchased liquids, etc.), the brand name, purchased location, whether e-cigarettes were shared with others. The nurse should act accordingly and report to the Department of Health [9]. 


\section{Conclusion}

Efforts from multiple agencies to address vaping has been insufficient. Of concern is that American youth continue to be exposed to the negative health effects of vaping. While the etiology of pulmonary injuries linked to electronic cigarette use remains unclear, the evidence has suggested that vitamin E acetate and THC inhalation have contributed to the US vaping death toll. We are fully aware of the importance of interdisciplinary and multifactorial approaches (e.g., education, nurse's involvement, regulatory changes) to address the vaping issue. It is imperative that nurses remain vigilant and be fully aware about the signs and symptoms of bronchiolitis obliterans. As outlined above, the nurse should direct patient to emergent care when the PCP is not available. Given the susceptibility of nicotine addiction in the youth and the current crisis, measures need to be taken to help prevent exposure, which in turn will prevent additional casualty vaping related death.

\section{References}

1. Duderstadt K (2015) E-Cigarettes: Youth and Trends in Vaping. Journal of Pediatric Health Care 29(6): 555-557.
2. Gonzalvo J, Constantine B, Shrock N, Vincent, A. (2016). Electronic Nicotine Delivery Systems and a Suggested Approach to Vaping Cessation. AADE in Practice 4(6): 38-42.

3. Schnur M (2019) Vaping Epidemic: A Public Health Crisis. Nurse Center Blog.

4. (2019) Vaporizers, E-Cigarettes, and other Electronic Nicotine Delivery Systems (ENDS) U.S. Food \& Drug Administration.

5. Miech R, Johnston L, O Malley P, M, Bachman JG, Patrick ME. (2019) Trends in Adolescent Vaping 2017-2019 The New England Journal of Medicine 381: 1490-1491.

6. Jatlaoui TC, Wiltz JL, Kabbani S, David A Siegel, Ram Koppaka, et al. (2019) Update: Interim Guidance for Health Care Providers for Managing Patients with Suspected E-cigarette, or Vaping, Product UseAssociated Lung Injury — United States, November 2019. Morbidity and Mortality Weekly Report (MMWR) 68: 1081-1086.

7. Milton CL (2018) Will Nursing Continue as the Most Trusted Profession? An Ethical Overview. Nursing Science Quarterly 31(1): 15-16.

8. CDC (2019) Outbreak of Lung Injury Associated with the Use of E-Cigarette, or Vaping, Products. Centers for Disease Control and prevention.

9. (2019) National Institute of Health office of dietary supplements. Vitamin E Fact Sheet for Health Professionals. 